\title{
Integral Sliding Mode Control for Improved Robustness and Accuracy of Induction Motors
}

\author{
Haider A. F. Mohamed, S. S. Yang, M. Moghavvemi \\ Centre for Research in Applied Electronics (CRAE), University of Malaya, 50603 Kuala Lumpur, \\ Malaysia (Tel: 603-7967-5370; e-mail: haider@um.edu.my).
}

\begin{abstract}
This paper proposes the use of sliding mode control to improve the performance of fieldoriented speed controllers of induction motors. The integral sliding mode control provides means to obtain an accurate estimate for disturbances and plant parameter perturbations. This estimator is used as an auxiliary control loop to reject these disturbances and parameter perturbations while avoiding chattering in the main control loop. The result is a controller with high degree of robustness and accuracy. Stability is achieved by using a design procedure based on a Lyapunov function. The feasibility of implementing such controllers is verified on a highly nonlinear system, an induction motor. Whether loaded or unloaded, the proposed integral sliding mode controller has proved to achieve high performance.
\end{abstract}

\section{INTRODUCTION}

Induction motors are popularly used in industrial applications that require motion because of their low cost, rugged structure, robustness, and maintainability. However, since the induction motor is basically a nonlinear coupling system, nonlinear control theory has been applied to the control of induction motors in many related research work. For example, Kim et al. (1990), Marino et al. (1993), Thomas et al. (1994) and Taylor (1994) utilized the feedback linearization design approach to achieve input-output decoupling, high dynamic performance, and high power efficiency. Furthermore, a feedback-linearisable system obtained by an integrator addition is presented in Chiasson (1993).

However, the parametric deviation will significantly affect dynamic performance and stability of practical implementation. Therefore, for the nonlinear feedback control of induction motors, many studies have also presented designs of compensators for the influence of the variation of motor parameters. For instance, Marino et al. (1993) and Thomas et al. (1994) presented an adaptive feedback linearization tracking controller for the induction motor. In these studies, an adaptation law is developed to compensate for the parametric uncertainty of the rotor resistance and the external load of induction motors. To regulate the electromagnetic torque, Ortega \& Espinosa (1993) and Ortega et al. (1993) developed an adaptive nonlinear feedback controller for tuning system parameters.

The control technique implemented in the above mentioned studies as well as in this work is the Field-Oriented Control (FOC) technique. The concepts of decoupling both flux and torque components in field-oriented control technique enable easier control of the induction motor. To drive the motor with the resulting current control signals, these currents are to be transformed to stator current components and then generated using pulse width modulated technique (PWM).
Nevertheless, as a result of the decoupling and the linearization required by the field-oriented control, precise knowledge of the motor parameters is required to yield a high performance controller. This requirement in practice is difficult to achieve due to several reasons. Firstly, the motor parameters used for the design of the controller are obtained through experiments or system identification methods where, in both, errors are very likely to appear depending on the overall conditions when carrying the measurement procedures. Secondly, even though the parameters obtained are precise values for the real system, these parameters vary when applying the control method due to change of the ambient temperature, change in the parameters due to the generated heat and change of the rotor position of the motor. In addition, it should be noted that the field-oriented control method uses a simplified motor model causing the control method to be an approximate and non-precise one.

To solve these problems, this paper suggests the use of sliding mode control method that reduces the calculation overhead used by the adaptive method (not to mention the convergence problem) to realize a robust control methodology that improves the efficiency of the conventional robust control method and also to eliminate the excitation of high-frequency unmodelled dynamics of the induction motor.

The proposed method works in conjunction with the fieldoriented method to improve the controller performance, eliminate the problems caused by parameter uncertainties, and reject the interference disturbances. It is based on the idea of composing the overall control action in two parts; a continuous part resulting from the field-oriented controller (PI) and a discontinuous part resulting from the sliding mode controller. The second part rejects disturbances and suppresses parametric uncertainties. 


\section{CONTROLLING THE INDUCTION MOTOR}

The plant discussed in this paper is a highly nonlinear induction motor. Its model described in the stator direct and quadrature frame $(\boldsymbol{d}-\boldsymbol{q})$ can be written as follows (Kubota \& Nakano, 1994):

$$
\begin{aligned}
& \frac{d \phi_{d}}{d t}=-\eta \phi_{d}+\eta M i_{d}, \quad \phi_{q}=0 \\
& \frac{d i_{d}}{d t}=\eta \beta \phi_{d}-\gamma i_{d}+\omega i_{q}+\frac{\eta M}{\phi_{d}} i_{q}^{2}+\frac{1}{\sigma L_{s}} u_{d} \\
& \frac{d i_{q}}{d t}=-\omega \beta \phi_{d}-\omega i_{d}-\gamma i_{q}+\frac{\eta M}{\phi_{d}} i_{d} i_{q}+\frac{1}{\sigma L_{s}} u_{q} \\
& \frac{d \omega}{d t}=\frac{T-T_{l}}{J}=\frac{1}{J}\left(\frac{N M}{L_{r}} \phi_{d} i_{q}-T_{l}\right)
\end{aligned}
$$

where:

$\eta=\frac{R_{r}}{L_{r}}, \sigma=1-\frac{M^{2}}{L_{s} L_{r}}, \beta=\frac{M}{\sigma L_{s} L_{r}}, \gamma=\frac{1}{\sigma L_{s}}\left(R_{s}+\frac{R_{r} M^{2}}{L_{r}^{2}}\right)$

The parameters are defined in Table 1.

Table 1. Induction motor parameter notations.

\begin{tabular}{|c|l|}
\hline Symbol & \multicolumn{1}{|c|}{ Parameter } \\
\hline$\phi_{d}, \phi_{q}$ & Rotor flux components in $(d-q)$ cords. \\
\hline$i_{d}, i_{q}$ & Stator currents in $(d-q)$ cords. \\
\hline$u_{d}, u_{q}$ & Stator voltages in $(d-q)$ cords. \\
\hline$L_{s}, L_{r}$ & Stator and rotor inductances \\
\hline$R_{s}, R_{r}$ & Stator and rotor resistances \\
\hline$T, T_{l}$ & Motor and load torques \\
\hline$M$ & Mutual inductance \\
\hline$N$ & Number of pole pairs \\
\hline$J$ & Moment of inertia \\
\hline$\omega$ & Electrical rotor speed \\
\hline
\end{tabular}

The induction motor is commonly controlled by the fieldoriented control scheme. The main idea is to transfer the three phase state variables to the stator frame and then to the two phase d-q coordinates reference frame locked to the rotor flux space vector in order to achieve decoupling between the motor flux and torque (Kubota \& Nakano, 1994). In this way, the motor variables can be separately controlled by stator direct-axis $(\boldsymbol{d})$ current and quadrature-axis $(\boldsymbol{q})$ current respectively, just as in a DC motor (Sen, 1990). Fig. 1 shows a general block diagram of an induction motor drive controlled by the FOC method. Here, the speed of the motor $\omega$ is measured and compared with the reference $\omega_{\text {ref }}$ to result in the speed error $e=\omega_{\text {ref }}-\omega$, which is processed by the speed controller to produce the control input command $i_{q_{-} r e f}$.
The transformation from the measured phase currents $i_{a}$ and $i_{b}$ to the d-q coordinates is shown in the following equations:

$i_{c}=-\left(i_{a}+i_{b}\right)$

$i_{\alpha}=\frac{2}{3} i_{a}-\frac{1}{3} i_{b}-\frac{1}{3} i_{c}$

$i_{\beta}=\frac{1}{\sqrt{3}} i_{b}-\frac{1}{\sqrt{3}} i_{c}$

and:

$i_{d}=i_{\alpha} \cos (\theta)-i_{\beta} \sin (\theta)$

$i_{q}=i_{\alpha} \sin (\theta)+i_{\beta} \cos (\theta)$

where $\theta$ is the rotor flux angle that can be obtained from the rotor speed $\omega$ and the slip frequency $\omega_{s l}$ is as follows:

$\theta=\int\left(\omega+\omega_{s l}\right) d t=\int\left(\omega+\eta M \frac{i_{q}}{\phi}\right) d t$

Equation (4) uses the flux $\phi$ as one of its parameters, and since the flux cannot be measured directly, a flux estimator is used. The flux estimator block of Fig. 1 is simply the Laplace transform of the first row in (1):

$\phi=\phi_{e s t}=\frac{\eta M}{\eta+s} i_{d}=\frac{M}{1+\tau \mathrm{S}} i_{d}$

where $\tau=1 / \eta=L_{r} / R_{r}$ is the time constant of the rotor.

Finally, the current references $i_{d_{-} \text {ref }}$ and $i_{q_{-} \text {ref }}$ are fed into the current controller blocks that generate the reference voltages $u_{d_{-} \text {ref }}$ and $u_{q_{-} \text {ref }}$.

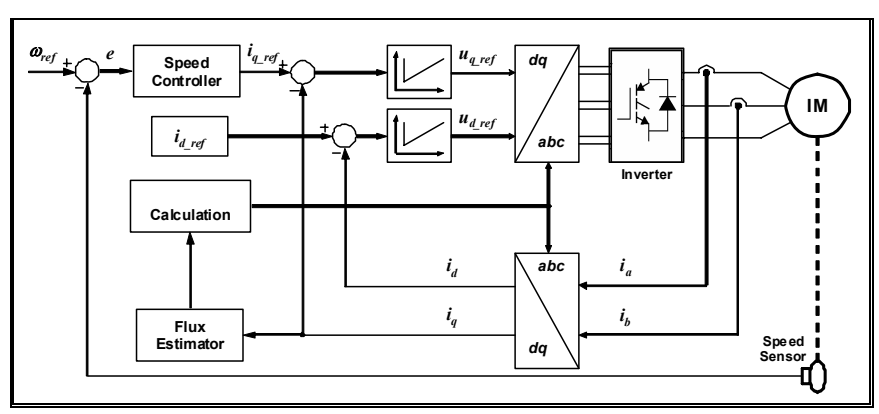

Fig. 1. FOC block diagram

\section{PROPOSED INTEGRAL SLIDING MODE CONTROL}

The main idea of disturbance rejection via sliding mode is to compose the two parts of the controller; the continuous and discontinuous components. The continuous component is used to control the overall behaviour of the system while the discontinuous component is used to reject disturbances and to suppress parametric uncertainties (Shi \& Lu, 1996, Utkin \& Shi, 1996, and Rios-Gastelum et al., 2003).

Basically, integral sliding mode control scheme provides a means to obtain an accurate disturbance and plant parameter perturbations estimation so that they can be removed from 
the system. This is done via an auxiliary control loop while avoiding chattering in the main control loop which normally consists of a continuous controller; a PI controller in this case.

A dynamic system under a closed loop control system can be represented by the following state-space equation:

$\dot{x}=f(x)+B(x) u+d(x, t)$

where $x$ is the state vector, $u$ is the control input vector and $d(x, t)$ is a function representing disturbances and perturbations caused by parameter variations and unmodelled dynamics.

To suppress $d(x, t)$ and hence obtain an accurate controller with high degree of robustness, design the control input as:

$u=u_{n m}+u_{d p}$

where $u_{n m}$ is the nominal control input while $u_{d p}$ is the introduced new control input vector that cancels the disturbances and perturbations to the system.

Now, (6) can be rewritten as:

$\dot{x}=f(x)+B(x) u_{n m}+B(x) u_{d p}+d(x, t)$

The condition for this design to work is to make the last two terms of (8) cancel each other, i.e.

$$
B(x) u_{d p}=-d(x, t)
$$

such that the closed loop control system follows the nominal design, $x(t)=x_{n m}(t)$, and hence all the disturbances and perturbations will no longer affect the system. Mathematically, (6) becomes:

$\dot{x}=f(x)+B(x) u_{n m}$

This proposed structure of this technique is achieved by replacing the speed controller of Fig. 1 with the combination shown in Fig. 2(a). The task now is to design the control input vector $u_{d p}$ using the sliding mode control technique. In

fact, the sliding mode controller here serves as a disturbance and perturbation identifier rather than a controller itself, while $u_{n m}$ is the vector control input vector designed using the nominal values of the system. First, design the sliding mode surface to be the disturbance and perturbation surface:

$s=s_{o}+z$

where

$s_{o}=\omega-\omega_{r e f}$

and $z(x, t)$ is the surface that needs to be designed to cancel disturbances and system parameter perturbations. For $z$ to satisfy (9), set the time derivative of $s$ equals to zero,

$$
\begin{aligned}
\dot{s} & =\dot{s}_{o}(x)+\dot{z}(x)=\frac{\partial s_{o}}{\partial x} \frac{\partial x}{\partial t}+\dot{z}(x)=0 \\
& =\frac{\partial s_{o}}{\partial x}\left\{f(x)+B(x) u_{n m}+B(x) u_{d p}+d(x, t)\right\}+\dot{z}(x)=0
\end{aligned}
$$

To ensure (9), $z$ needs to be designed as:

$$
\dot{z}(x)=-\frac{\partial s_{o}}{\partial x}\left\{f(x)+B(x) u_{n m}\right\}, z(0)=-s_{o}(x(0))
$$

Therefore, substituting from (11) and (12), and using state equations (1) to substitute the sate derivative, the following is obtained:

$$
\begin{aligned}
& \dot{z}=\frac{d \omega_{\text {qref }}}{d t}-\frac{N M_{n m} \phi_{d}}{J_{n m} L_{r_{-} n m}} i_{q_{-} n m}+\frac{T_{l_{-} n m}}{J_{n m}}, \\
& z(0)=\omega_{\text {qref }}(0)-\omega(0)
\end{aligned}
$$

where the subscript $\mathrm{nm}$ denotes the nominal design values that are used for the vector controller. The second stage of the design is the discontinuous control law which is as follows:

$u_{d p}=i_{q_{-} d p}=-g \operatorname{sign}(s)$

where $g$ is a constant control gain.

To obtain the gain value $g$, design a Lyapunov function $V_{I S M C}$ as:

$V_{I S M C}=\frac{1}{2} s^{2}$

For the sliding mode to be enforced in the surface $s=0$, the time derivative of (17) should be negative definite, i.e.

$\dot{V}_{I S M C}=s \dot{s}<0$

First, calculate $\dot{S}$ by the substitution of current derivative values from (1) into the time derivative of (11) and (12) and using (7) and (15) for the values of $u_{n m}$ ( $i_{q_{-} n m}$ in our case) and $\dot{z}$ yields:

$\dot{s}=\dot{s}_{o}+\dot{z}=\frac{N M \phi_{d}}{J L_{r}} i_{q_{-} d p}+\varepsilon \phi_{d} i_{q_{-} n m}+\varepsilon_{l}$

with the perturbation errors,

$\varepsilon=\frac{N M}{J L_{r}}-\frac{N M_{n m}}{J_{n m} L_{r_{-} n m}} \quad$ and $\quad \varepsilon_{l}=\frac{T_{l_{-} n m}}{J_{n m}}-\frac{T_{l}}{J}$

By substituting (16) and (19) into (17) one can obtain the gain value $g$;

$-\frac{N M \phi_{d}}{J L_{r}} g \operatorname{sign}(s)+\varepsilon \phi_{d} i_{q_{-} n m}+\varepsilon_{l}<0$

which yield, with the use of (7), to the following condition after taking into account the maximum change in parameter variations :

$g>\max \left\{\frac{J L_{r}}{N M \phi_{d}}\left|\varepsilon \phi_{d} i_{q_{-} n m}-\varepsilon_{l}\right|\right\}$ 


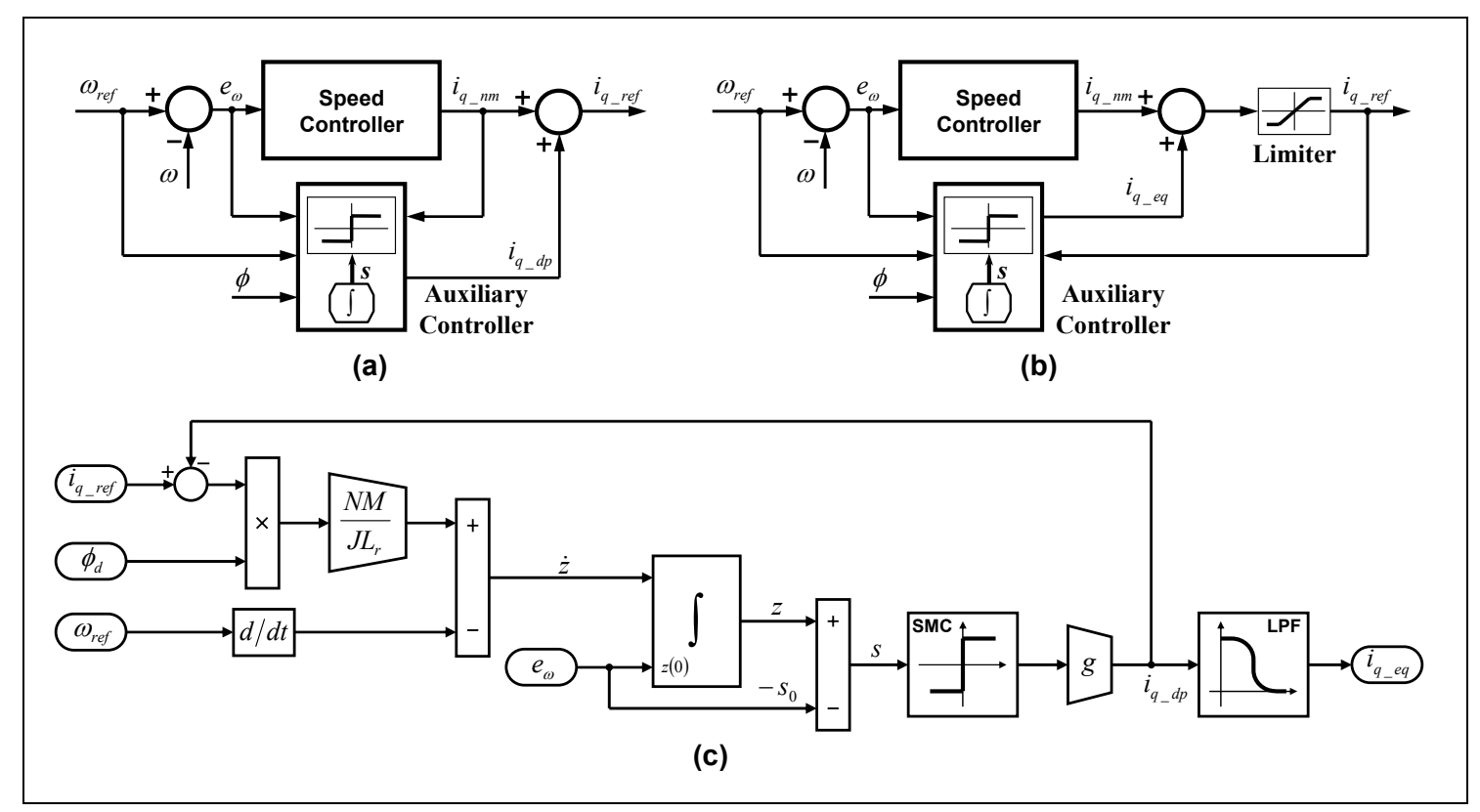

Fig. 2. Proposed integral sliding mode control method: (a) the original structure of integral sliding mode control, (b) the modified integral sliding mode control block diagram, and (c) the auxiliary controller block diagram.

Remark 1: The initial condition of $z$ in (14) ensures that the initial condition of the sliding manifold $S$ equals to zero; $s(0)=0$. In other words, sliding mode is to occur starting from the initial time instant.

Remark 2: Ideally, the switching of control occurs at infinitely high frequency to eliminate the deviations from sliding manifolds (Utkin, 1993, and Cupertino et al., 2000). In practice, the switching frequency is not infinitely high due to the finite switching time. Combined with the effects of unmodelled dynamics, this finite switching causes undesired chattering of the control. For this reason, $i_{q_{-} d p}$ is replaced by the equivalent control input $i_{q_{-} e q}$ through a low pass filter with a cutting frequency carefully chosen faster than the perturbations and disturbances dynamics, but also slow enough not to excite any unmodelled dynamics (Utkin, 1972, and Hung et al., 1993).

To illustrate the possibility of such a replacement, consider (13) which implies that the equivalent control is the solution to $\dot{s}=0$ with respect to $i_{q_{-} d p}$ under condition $s=0$. Thus, by using (19), the solution yields:

$i_{q_{-} e q}=\left(i_{q_{-} d p}\right)_{e q}=-\frac{J L_{r}}{N M \phi_{d}}\left(\varepsilon \phi_{d} i_{q_{-} n m}+\varepsilon_{l}\right)$

Hence, the equivalent control $i_{q e q}$ that can be extracted from $i_{q_{-} d p}$ using a low-pass filter compensates exactly for system disturbances and parameter perturbations.

Remark 3: Since all the controllers implemented in this work use a limiter to limit the current command $i_{q_{-} \text {ref }}$, the proposed integral sliding mode controller is modified in the following manner; (7) is used to modify $i_{q_{-} n m}$ in (15) to result in the following equation:

$\dot{z}=\frac{d \omega_{\text {qref }}}{d t}-\frac{N M_{n m} \phi_{d}}{J_{n m} L_{r_{-} n m}}\left(i_{q_{-} r e f}-i_{q_{-} d p}\right)+\frac{T_{l_{-} n m}}{J_{n m}}$,

$z(0)=-s_{o}(0)=\omega_{\text {qref }}(0)-\omega(0)$

This modification in the control structure is shown in Fig. 2(b). The proposed auxiliary control block diagram of (24) is illustrated in Fig. 2(c).

\section{REAL-TIME IMPLEMENTATION}

The hardware experimental setup uses a DSP kit based on TMS320LF2407 that has all the peripherals needed to control a 3-phase AC motor. It also contains all the A/D converters needed to read the currents of the motor. Two counters of the DSP kit are used to perform the necessary interrupt service routines (ISRs) for the speed and current controls. The feedback control system application based on the TMS320LF2407 DSP kit uses the two timers of the DSP in the following manner:

Timer 1 is programmed to generate a $20 \mathrm{kHz}$ PWM. The same timer is also used for real-time interrupt generation in order to activate the fast and slow interrupts for current and speed control implementation respectively. For a $20 \mathrm{kHz}$ PWM frequency, this gives an interrupt every 50 microseconds. Using a software counter, the current control routine is executed every two PWM periods (i.e. each 100 microseconds). Another software counter is used to execute the speed control routine every twenty PWM periods (i.e. each $1 \mathrm{~ms})$.

Timer 2 counts the encoder pulses. The code uses this information to compute the motor speed as an encoder 
position difference over one sampling period of the speed control loop.

The induction motor used in this paper is a $0.37 \mathrm{~kW}$ squirrel cage induction motor with the parameters shown in Table 2.

\section{Table 2. Induction motor parameter notations.}

\begin{tabular}{|c|c|}
\hline Parameter & Value \\
\hline$L_{s}=L_{r}$ & $1.52 \mathrm{H}$ \\
\hline$R_{s}$ & $24.6 \Omega$ \\
\hline$R_{r}$ & $16.1 \Omega$ \\
\hline$M$ & $1.46 \mathrm{H}$ \\
\hline$N$ & $1(2$ poles $)$ \\
\hline$J$ & $5.610^{-3} \mathrm{~kg} \cdot \mathrm{m}^{2}$ \\
\hline
\end{tabular}

The first test is performed on the motor in the nominal design conditions. A 2000rpm step reference signal is fed to the system under both vector control and integral sliding mode control methods. The results are shown in Fig. 3.

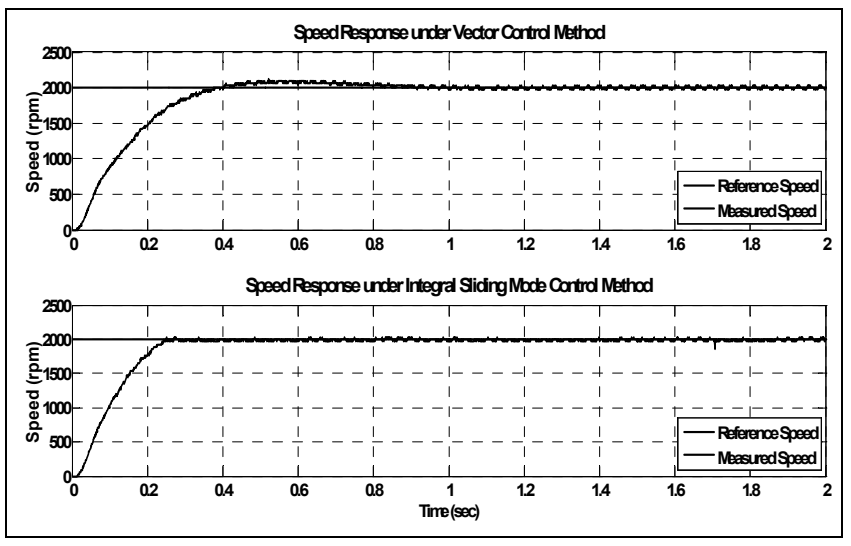

Fig. 3. Speed step-response under both VC and ISMC.

The speed response of the vector control real-time implementation has a rise time of $t_{r}=0.38 \mathrm{sec}$ and settling time of $t_{s}=0.75 \mathrm{sec}$ under the $2 \%$ allowable tolerance criteria. The response of the motor exhibits about 100rpm overshoot, or $5 \%$ overshoot. The system, however, suffers from small fluctuations around the reference in steady state due to noise and relatively low accuracy of the optical encoder that measures the speed.

As for the proposed scheme, the results illustrate an enhanced transient response of the system as no overshoot occurs. This makes the proposed scheme even faster than the vector control method. Here, the rise time is $t_{r}=0.25 \mathrm{sec}$ and the settling time is $t_{s}=0.24 \mathrm{sec}$.

To investigate the properties of the proposed ISMC method, the two sliding manifolds $z$ and $s_{o}$ and the auxiliary controller action are recorded in Fig. 4. As stated in Remark 1, it can be seen in Fig. 4 that the sliding mode occurs from the initial state $t=0 \mathrm{sec}$. This control action is averaged using the low-pass filter to obtain the equivalent control, thus minimizing the chattering effect as discussed in Remark 2.

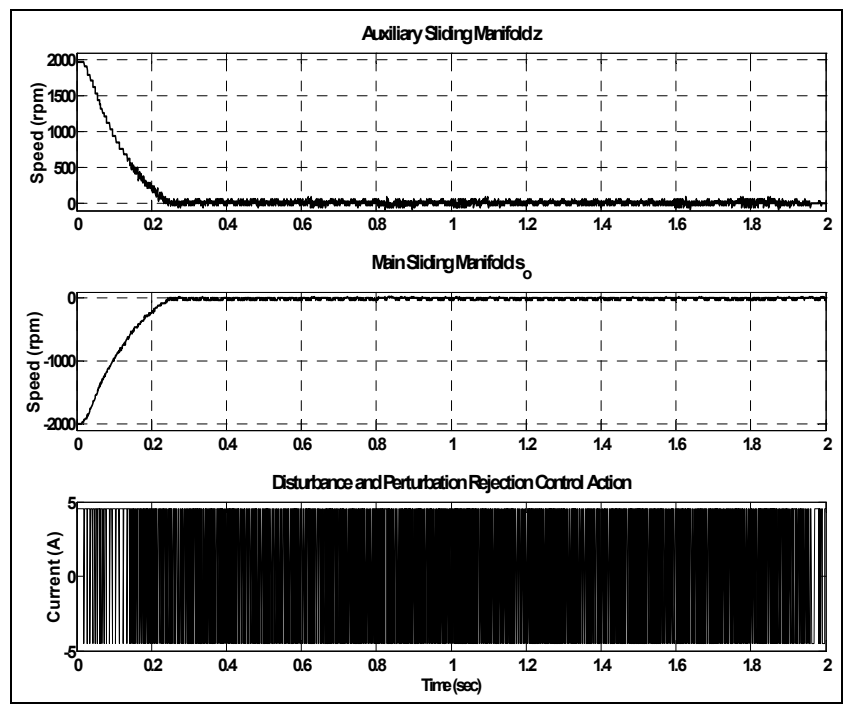

Fig. 4. Auxiliary manifold $z$, sliding manifold $s_{o}$ and auxiliary control action $i_{q_{-} d p}$.

The purpose of the second test is to investigate the performance of the proposed control method under system parameter perturbations. Since it is not possible to modify the rotor's parameters of a squirrel cage induction motor, the test is carried out by modifying the parameters of the continuous controller (PI) in two cases; over-damped (Case 1) and underdamped (Case 2). The test also examines the system under load torque conditions; a load torque with the value of $4.5 \mathrm{~N} . \mathrm{m}$. is suddenly applied to the shaft of the motor at $t=1.5 \mathrm{sec}$ when the system is at steady state. The two controllers' real time implementation results are recorded and shown in Figs. 5, 6 and 7 for the system in nominal design conditions, Case 1 and Case 2 respectively.

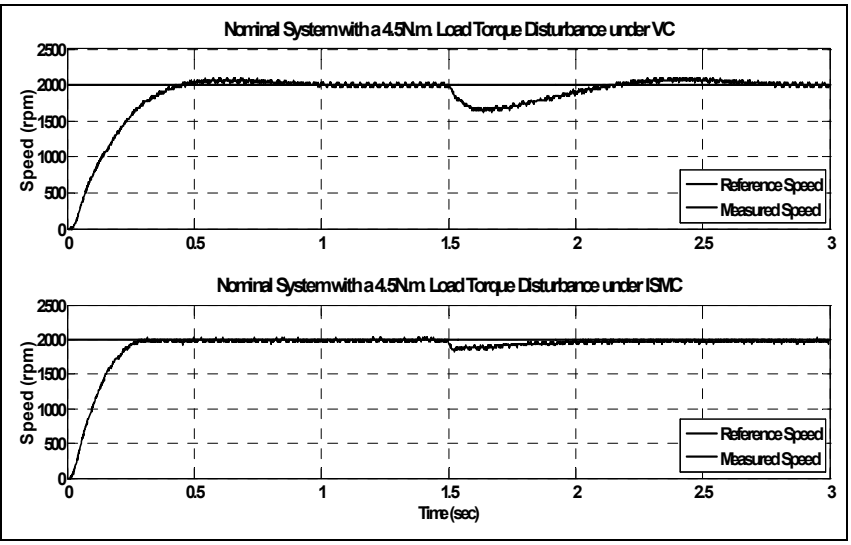

Fig. 5. VC and ISMC speed response of nominal system under $4.5 \mathrm{~N} . \mathrm{m}$. load torque disturbance.

As can be seen from the results, the proposed control method always has faster transient responses and settling times 
without any overshoots. In addition, the integral sliding mode control method reduces the drop caused by the applied load torque and results in a faster recovery time. Thus, it enhances the performance of the system in the face of system parameter perturbations and disturbances.

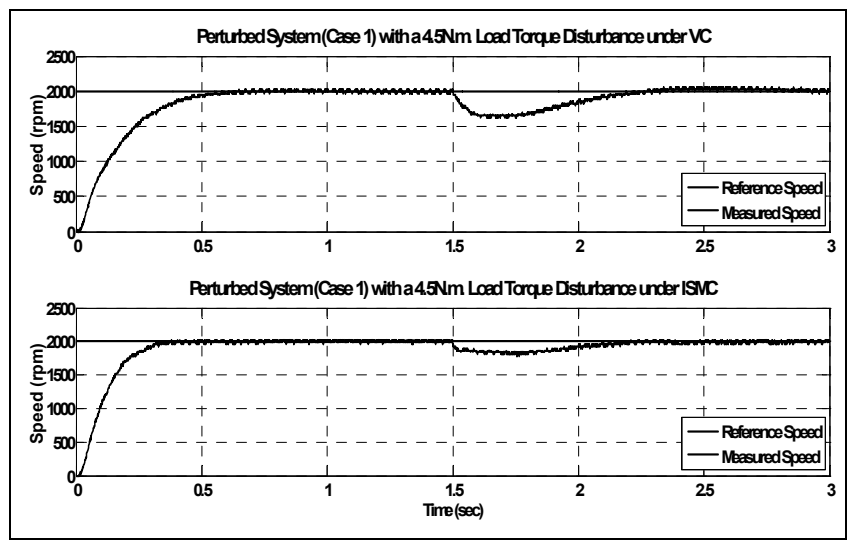

Fig. 6. VC and ISMC speed response of perturbed system (Case 1) under 4.5N.m. load torque disturbance.

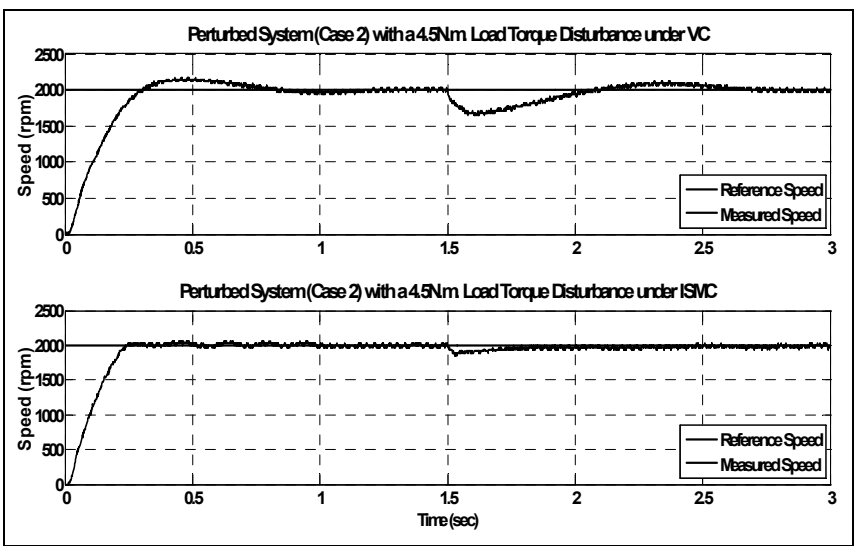

Fig. 7. VC and ISMC speed response of perturbed system (Case 2) under 4.5N.m. load torque disturbance.

\section{CONCLUSIONS}

This work has proposed the use of a sliding mode controller to compensate for system parameter perturbations and external disturbances which occur in speed control of induction motors under the field-oriented control method. The new design is implemented, tested and compared to a conventional vector controller. The real-time implementations were carried out under nominal design conditions, perturbed system conditions and applied load torque conditions. From the results, the proposed integral sliding mode controller rejected the system parameter perturbations and disturbances well compared to the conventional vector control method. Furthermore, the proposed scheme illustrated faster rise times, settling times and recovery times. The overshoot of the vector control method was cancelled out and the load drops were minimised.
In conclusion, the proposed design yields a robust control system since the compensation starts even at the starting of the system. Industrial applications that use induction motors and require fast responses and high accuracy can greatly benefit from the proposed control scheme.

\section{REFERENCES}

Chiasson J. (1993). Dynamic feedback linearization of the induction motor, IEEE Trans. Automatic Control, Vol. 38, pp. 1588-1594.

Cupertino F., Lattanzi A. and Salvatore L. (2000). Sliding mode control of an induction motor, IEE Power Electronics and Variable Speed Drives Conference, No. 475, pp. 206-211.

Hung J. Y., Gao W. and Hung J. C. (1993). Variable structure control: a survey, IEEE Trans. Industry Applications, Vol. 40, pp. 2-21.

Kim D. I., Ha I. J. and Ko M. S. (1990). Control of induction motors via feedback linearization with input-output decoupling. Int. Journal Control, Vol. 51(4), pp. 863883.

Kubota H., Matsuse K. and Nakano T. (1994). DSP-based speed adaptive flux observer for induction motor, IEEE Trans. Automatic Control, Vol. 2, pp. 380-384.

Marino R., Peresasa S. and Valigi P. (1993). Adaptive inputoutput linearizing control of induction motors, IEEE Trans. Automatic Control, Vol. 38, pp. 208-221.

Ortega R. and Espinosa G. (1993) Torque regulation of induction motors, Automatica, Vol. 29(3), pp. 621-633.

Ortega R., Canudas C. and Seleme S. (1993). Nonlinear control of induction motors: Torque tracking with unknown load disturbance, IEEE Trans. Automatic Control, Vol. 38, pp. 1675-1680.

Rios-Gastelum O.G., Castillo-Toledo B. and Loukianov A.G. (2003). Nonlinear block integral sliding mode control: application to induction motor control, IEEE Proc. Decision and Control, Vol. 3, pp. 3124-3129.

Sen P. C. (1990). Electric motor drives and control: past, present and future, IEEE Trans. Industrial Electronics, Vol. 37, pp. 565-575.

Shi J. and Lu Y. S. (1996). Chatter free variable structure perturbation estimator on the torque control of flexible robot joints with disturbance and parametric uncertainties, IEEE Proc. Industrial Electronics, Control, and Instrumentation, Vol. 1, pp. 238-243.

Taylor D. G. (1994). Nonlinear control of electric machines: An overview, IEEE Contr. Syst. Mag., Vol. 14, pp. 4151.

Thomas V. R., Jean M. D. and Luc D. (1993). Adaptive nonlinear speed control of induction motors, Int. Journal Adapt. Control Signal Process., Vol. 7(5), pp. 435-455.

Utkin V. (1972). Equations of slipping regimes in discountinuous systems II, Automation and Remote Control, Vol. 33, pp. 211-219.

Utkin V. I. (1993). Sliding mode control design principles and applications to electric drives, IEEE Trans. Industrial Electronics, Vol. 40, pp. 23-36.

Utkin V. and Shi J. (1996). Integral sliding mode in systems operating under uncertainty conditions, IEEE Proc. Decision and Control, Vol. 4, pp. 4591-4596. 